\title{
LA FORMACIÓN DEL MAGISTERIO RURAL EN ENTRE RÍOS: DEMANDAS PRODUCTIVAS Y ASPIRACIONES PROFESIONALES (1914-1930)
}

\author{
Janet Priscila Cian* \\ IRICE-CONICET, Universidad Nacional de Rosario, Argentina \\ janetcian@hotmail.com
}

Recibido: 16/08/2019 Aceptado: 30/11/2019

\begin{abstract}
Resumen
En este artículo se reconstruye la política educativa para la formación del magisterio rural desenvuelta en la provincia de Entre Ríos durante los gobiernos de la Unión Cívica Radical que transcurren desde fines de 1914 a 1930. Se sostiene que durante dicho período se dio continuidad a la formación del magisterio rural mediante los cursos temporarios y la Escuela Normal Rural provincial, que comenzaron en 1903. Las modificaciones constatadas en ese lapso implicaron una mayor atención a la preparación pedagógica, en detrimento de la centralidad que tuvo la formación agropecuaria en sus primeros años. En esta reorientación confluyeron la particular articulación de la administración provincial, las agencias encargadas de la capacitación agropecuaria y el inicio de las regulaciones sobre el trabajo docente. Se trabaja desde una metodología cualitativa que combina aportes de la historiografía de las instituciones educativas con los recientes estudios sobre el Estado.
\end{abstract}

Palabras clave: Educación rural - Escuela Normal Rural - Políticas educativas - Cursos Temporarios - Unión Cívica Radical.

\footnotetext{
Abstract

This article reconstructs the educational policy for the formation of the rural magisterium developed in the province of Entre Ríos during the governments of the Radical Civic Union that took place from the end of 1914 to 1930. It is argued that during this period the training continued the rural teaching through temporary courses and the Provincial Rural Normal School, which began in 1903. The modifications observed in that period implied more attention to the pedagogical preparation, in detriment of the centrality that the agricultural training had in its early years. In this reorientation the particular articulation of the provincial administration, the agencies in charge of

* Becaria doctoral del Consejo Nacional de Investigaciones Científicas y Técnicas de Argentina (CONICET), Jefa de Trabajos Prácticos en Sociologia de la Educación y Auxiliar docente Historia de la Educación. Con dependencia en la Facultad de Ciencias de la Educación (UNER) y en la Facultad de Humanidades, Artes y Ciencias Sociales (UADER).
} 
REVISTA DE LA ESCUELA DE CIENCIAS DE LA EdUCACIÓN, AÑO 16, NRO. 15, VOL. 2, JULIO A DICIEMRE DE 2020. PÁGINAS 143-154. ISSN 2362-3349 (EN LÍNEA). LA FORMACIÓN DEL MAGISTERIO RURAL EN ENTRE RÍOS: DEMANDAS PRODUCTIVAS Y ASPIRACIONES PROFESIONALES (1914-1930). JANET PRISCILA CIAN

agricultural training and the beginning of the regulations on teaching work came together. This paper follows a qualitative methodology that combines contributions of the historiography of educational institutions with recent studies on the State.

Keywords: Rural education - Normal rural school - Educational policies - Temporary courses Radical civic union.

\section{Introducción}

Los resultados que arrojó el Tercer Censo Nacional de la República Argentina, realizado en 1914, resumen los cambios experimentados en el país durante un período relativamente corto. En el plano demográfico, la población total estaba constituida por unos 7.905.502 habitantes ${ }^{1}$, mostrando el impacto del arribo de la inmigración de ultramar (Devoto, 2009; Cattaruzza, 2009). Otros datos relevantes fueron: el incremento de la producción agropecuaria, manifiesto en el volumen de las exportaciones locales, en comparación con otros países latinoamericanos ${ }^{2}$ (Barsky y Gelman, 2005; Hora, 2010; Míguez, 2011), la construcción de la red ferroviaria y las inversiones externas realizadas en esa etapa (Regalsky, 1986). La provincia de Entre Ríos, localizada dentro del área de expansión agraria de la pampa húmeda, también formó parte del área donde se percibió el salto poblacional registrado entre los censos de 1895 y 1914. Si bien a partir del primero ya se había comenzado a evidenciar un proceso de decrecimiento de la población ubicada en el medio rural ${ }^{3}$, que se profundizaría durante gran parte del siglo XX (Mateo et. al, 2017), el censo de 1914 mostraba cierta continuidad en la tendencia, aunque la mayor proporción de la población aún seguía radicada en este espacio. El crecimiento del área cultivada también fue significativo -ese año, había un total de 794.476 hectáreas de cereales y forrajeras, duplicando el registro del censo de 1895- aunque representaba una extensión menor en comparación con otras provincias de la región pampeana. También la ganadería ocupaba un lugar preponderante como actividad económica y en cuanto a la ocupación del territorio. El inicio de la Primera Guerra Mundial desaceleró el crecimiento de la economía agroexportadora registrado entre 1880 y 1914 a escala nacional, situación de la cual Entre Ríos no estuvo exenta. A las dificultades impuestas por el conflicto bélico, se agregaron los efectos de una alicaída economía provincial en crisis desde 1912 y el cambio político en la administración provincial, al iniciarse la extensa e ininterrumpida etapa de gobiernos presididos por la Unión Cívica Radicall que se extendería hasta 1943.

Estos datos sobre las características demográficas y económicas del país y, en particular, de la provincia permiten observar la importancia que tenía el espacio rural durante las primeras décadas del siglo XX, no obstante el estudio de la escolarización de la población radicada en el este medio registra escasa atención de la historiografía de la educación. Recién a comienzos del actual milenio ha comenzado a modificarse esta tendencia, con investigaciones que se han focalizado, en su mayoría, en la región pampeana, durante la primera mitad de ese siglo (Ascolani, 2012, 2015, 2017; Gutiérrez, 2005, 2007; Petitti, 2016), aunque también existen algunos trabajos sobre el área patagónica (Pierini, 2016). En este sentido, un diagnóstico análogo se puede efectuar de las investigaciones que examinan la profesionalización del magisterio, las prácticas escolares y extraescolares producidas en este ámbito, soslayadas ante el interés de explorar la conformación institucional de las Escuelas Normales Nacionales, las corrientes pedagógicas que impulsaron, las trayectorias de funcionarios egresados de las mismas, entre algunos de las problemáticas que han suscitado mayor atención (Carli, 1993; Fiorucci, 2014; Ascolani, 2018). Si bien este trabajo no pretende tratar en particular tales ausencias, se puede constatar que la renovación de las investigaciones que toman como objeto el Estado y las políticas públicas desarrolladas en los últimos años han posibilitado la emergencia de investigaciones que discuten las escalas de análisis tradicionales (Palermo y Silva, 2016) y reconocen la vacancia de estudios a escala subnacional, una sentida demanda para la comprensión de la configuración estatal en cada territorio (Bohoslavsky, y Soprano, 2010; Pereyra, 2012; Di Liscia y Soprano, 2017). Con estas

\footnotetext{
${ }^{1}$ El Censo Nacional de 1895 mostró un total de población de 3.955.110 habitantes. Los datos de 1914 presentaban una tasa de crecimiento de un 3,1\%, el doble del resto de Latinoamérica

${ }^{2}$ El resto de los países latinoamericanos multiplicaban sus exportaciones por 7,3 veces y la Argentina lo hacía por 45,2.

${ }^{3}$ En 1914, el total de la población de Entre Ríos era de 425.373. En 1895 se habían relevado 291.019 habitantes.

${ }^{4}$ El primer golpe de Estado de la Argentina, realizado el 6 de septiembre de 1930, y que derivó en la intervención federal de los gobiernos provinciales exceptuó a la provincia de San Luis y Entre Ríos. En esta última, la UCR gobernaba desde 1914 y se caracterizó por su oposición a la figura de Hipólito Yrigoyen, conformando el sector denominado impersonalista (Pereira, 2018).
} 
REVISTA DE LA ESCUELA DE CIENCIAS DE LA EdUCACIÓN, AÑO 16, NRO. 15, VOL. 2, JULIO A DICIEMRE DE 2020. PÁGINAS 143-154. ISSN 2362-3349 (EN LÍNEA). LA FORMACIÓN DEL MAGISTERIO RURAL EN ENTRE RÍOS: DEMANDAS PRODUCTIVAS Y ASPIRACIONES PROFESIONALES (1914-1930). JANET PRISCILA CIAN

indagaciones, se ha comenzado a examinar las políticas públicas generadas por los Estados provinciales, la organización de sus agencias y burocracias locales.

En lo referido a la formación profesional del magisterio rural con un currículo que incluyera contenidos agropecuarios y orientado a atender demandas productivas locales, investigaciones previas visibilizaron las creaciones institucionales realizadas desde el Estado provincial en Entre Ríos (Gutiérrez, 2007; Ascolani, 2007; Mayer, 2014). Desde inicios del siglo XX, algunos funcionarios provinciales impulsaron políticas públicas que intentaban articular cierto intento de distribución de la tierra mediante la creación de nuevas colonias agrícolas y la capacitación agrotécnica de los jóvenes para contribuir al mejoramiento agropecuario. De esta forma, la organización de una Escuela Normal Rural ${ }^{5}$ de carácter provincial, en 1904, no representó una experiencia aislada, ya que se conectaba con la creación de escuelas agropecuarias repartidas en cada departamento de la provincia ${ }^{6}$ que prepararían un personal técnico idóneo necesario para impulsar el mejoramiento agropecuario. Estas instituciones fueron establecidas a partir de la Constitución provincial de $1903^{7}$ bajo la denominación de "educación especial" y tuvieron apoyo político y financiero durante el lustro que se extendió hasta inicios de 1909 (Cian, 2018).

La posibilidad de profesionalización del magisterio rural provincial que ofrecía la creación de una Escuela Normal Rural se articuló con una política de capacitación de los maestros en ejercicio y aspirantes sin título por medio de los cursos temporarios, iniciados en 1903. Con una duración de dos veranos, se daba una capacitación elemental que incluía contenidos agropecuarios para los maestros en ejercicio, muchos de ellos provenientes de las comunidades inmigrantes, y para los aspirantes a maestros. La habilitación obtenida luego de los exámenes les permitía ejercer el magisterio en el medio rural y les proporcionaba el derecho al ascenso en el mismo medio. Concebida como una política coyuntural que funcionaría de forma descentralizada en las ciudades cabeceras de los principales departamentos y que culminaría con la apertura de la Escuela Normal Rural, los cursos para varones se mantuvieron y se institucionalizaron en dicha escuela a partir de 1907. Para las mujeres, en cambio, se habilitaron mesas examinadoras en algunas ciudades, en las que también podían presentarse los varones ${ }^{8}$

En el caso de la Escuela Normal Rural, comenzó a funcionar en 1904, en un extenso campo que contaba con infraestructura. La meta de establecer secciones productivas que pudieran impulsar producciones locales, entre las que se encontraba la avicultura y la cremería, y la organización de un curriculum que combinaba la formación profesional del magisterio y contenidos agropecuarios suscitaron numerosas dificultades que han sido documentados la prensa local y los expedientes institucionales. A esto se sumaba el alto financiamiento que demandaba sostener la enseñanza agropecuaria, así como los conflictos con otras dependencias del Estado provincial respecto a los medios de modernizar el agro, y los desacuerdos entre funcionarios educativos nacionales y provinciales por las tasas de analfabetismo. Este último dio lugar a la realización de cambios en la política educativa a partir de 1909, de las que resultó exenta la Escuela Normal Rural. Los reiterados conflictos entre profesores y técnicos agrónomo, y las discordias en torno a la administración de los fondos producidos derivaron en la intervención del Consejo General de Educación (en adelante CGE), en 1912, que dispuso la reorganización y traslado del personal directivo, cambios en la normativa interna y en el desarrollo curricular, además de la reducción de la extensión de tierras para la experimentación agrícola.

En este artículo se reconstruyen, en primer lugar, las políticas educativas en la coyuntura comprendida entre 1914 y 1921, considerando aspectos referidos a la profesionalización del magisterio rural, el funcionamiento de la Normal Rural y los inicios de la legislación sobre estabilidad docente. Luego, se examina la etapa posterior, hasta 1930, observando las tensiones entre el aumento de la oferta de maestros, la expansión de la obligatoriedad escolar y las altas tasas de analfabetismo. Se sostiene como hipótesis que la política de profesionalización del magisterio rural se efectuó mediante la Escuela Normal Rural y los cursos temporarios, a la vez que las modificaciones curriculares y de la formación de los aspirantes tuvieron como objetivo consolidar una mayor orientación pedagógica en detrimento de la capacitación agropecuaria. Esto tuvo relación con cierta reorientación en las política públicas para el mejoramiento del sector agropecuario, ya que el destacado espacio que ocupó el CGE en un interregno de la etapa previa fue progresivamente ocupado

\footnotetext{
${ }^{5}$ Se ubicó a 20 kilómetros de la ciudad de Paraná, en Tezanos Pinto, que en esa etapa pertenecía al departamento Diamante.

${ }^{6}$ Sólo se lograron concretar: la escuela agropecuaria e industrial "Tomás Espora" (1904) en Concordia, la agropecuaria "9 de julio" (1904) en Nogoyá, Agropecuaria “Justo José de Urquiza” (1905) en Villaguay. Completaba este cuadro la escuela Nacional de Agricultura y Ganadería "Las Delicias" (1900) y agropecuaria de Villa Urquiza (1896).

${ }_{8}^{7}$ Los cambios fueron presentados en la Ley de Educación provincial № 1905, de 1904.

${ }^{8}$ La mayor presencia femenina y los resultados poco satisfactorios de las mesas examinadoras conllevaron la reapertura de los Cursos Temporarios para mujeres para 1913-1914.
} 
REVISTA DE LA ESCUELA DE CIENCIAS DE LA EdUCACIÓN, AÑO 16, NRO. 15, VOL. 2, JULIO A DICIEMRE DE 2020. PÁGINAS 143-154. ISSN 2362-3349 (EN LÍNEA). LA FORMACIÓN DEL MAGISTERIO RURAL EN ENTRE RÍOS: DEMANDAS PRODUCTIVAS Y ASPIRACIONES PROFESIONALES (1914-1930). JANET PRISCILA CIAN

por el Departamento de Agricultura ${ }^{9}$, que desarrolló una acción extraescolar (Cian, 2019). La preparación profesional de los maestros se articuló con la regulación del trabajo docente, que se comenzó a institucionalizar mediante la Ley de ingreso, ascenso y estabilidad del magisterio. Se analiza un corpus variado, compuesto por: boletines y memorias de educación del CGE de Entre Ríos; artículos periodísticos; leyes, decretos y debates parlamentarios; copiadores y correspondencia del archivo institucional de la Escuela "Juan Bautista Alberdi".

\section{Los ejes de debate educativo: analfabetismo, profesionalización y estabilidad del magisterio provincial}

En el año 1932, el Ministro de Hacienda, Justicia, Culto e Instrucción Pública advertía, en el mensaje para la aprobación del presupuesto provincial, la necesidad de revisar la situación de la Escuela Normal Rural "Juan Bautista Alberdi", considerada "un intento bien pensado en la teoría, pero malogrado en la práctica", debido a lo oneroso de su mantenimiento y el escaso impacto que tenían sus egresados, los cuales no trabajaban en el medio rural, sino que "...aspiran a continuar estudios universitarios o trabajar en centros urbanos. Motivos suficientes para mostrar el fracaso de este proyecto" (Provincia de Entre Ríos, 1932, p. 65). La grave crisis económica general que afectaba a la economía nacional obligaba, en el escenario provincial, a la reducción de gastos, incluyendo los que insumían el mantenimiento de esta escuela. Estos cuestionamientos a la institución no resultaban novedosos, ya que formaban parte de los argumentos críticos de quienes objetaron el establecimiento desde sus inicios. En estas críticas habían coincidido tanto algunos sectores del oficialismo -el Partido Autonomista Nacional-, así como sus opositores. A pesar de esto, la institución logró organizarse y tuvo una relativa estabilidad durante los gobiernos de la Unión Cívica Radical, iniciados a fines de 1914.

La sanción en 1912 de la Ley № 8.871, de voto secreto y obligatorio -según padrón militar para varones- tuvo entre sus primeros ensayos las elecciones provinciales realizadas el 29 de julio de 1914, en las que triunfó la formula radical compuesta por Miguel Laurencena y Luis Etchevehere, electos como gobernador y vicegobernador, respectivamente. El recambio de autoridades políticas implicó la renuncia del elenco de funcionarios de la administración provincial entre los que se encontraba Manuel Pacifico Antequeda, presidente del CGE desde 1903, que había fomentado la formación normalista. En su lugar asumió otro maestro normal, Alfredo Villalba, cuya primera iniciativa fue proponer declarar en comisión de todo el personal docente de la provincia, en concordancia con lo dispuesto para el resto de la administración pública. La disposición fue rechazada por algunos vocales del CGE, que llegaron a considerarla análoga a un estado de sitio: "medida suprema ante la cual quedan en suspenso todas las garantías constitucionales". Entre los argumentos que esgrimían para su cuestionamiento, se encontraba la garantía que ofrecía un título, una posesión que los distinguía del empleado común. De esta forma, se hacían eco de un reclamo del magisterio local en torno a la estabilidad y ascenso de este personal que había contado con el aval del radicalismo local, en su etapa de oposición al régimen conservador. Finalmente, la iniciativa de declaración en comisión no prosperó, pero hubo restricciones económicas. En el presupuesto para 1915, fueron suprimidas las partidas para la compra de herramientas y útiles agrícolas para uso escolar, también el dictado de los cursos temporarios y gastos para festejo de las fiestas patrias. A esto se agregaba una deuda en pago de salarios a los maestros provinciales de más de cinco meses. Otra situación que generaba malestar era las mesas examinadoras para la obtención del título de maestro rural no estaban habilitadas.

El recambio de funcionarios también derivó en un exhaustivo examen de las estadísticas educativas oficiales, que ya habían sido objeto de controversias en la etapa anterior (Ossanna y Hadad, 2011). Las altas tasas de analfabetismo que registraban los informes contrastaban con el $12 \%$ arrojado por el censo provincial de 1910, y con el 18,87\%, resultante del Censo Nacional de 1914, en un contexto de aumento del número de escuelas y disminución de la población escolar (Provincia de Entre Ríos, 1919, p. 12). También se cuestionaron los datos sobre los maestros en ejercicio -muchos de los cuales ocupaban otros puestos en la administración- y sobre la cantidad de maestros titulados que se registraba. La categoría "diplomado" comprendía una diversidad de situaciones: estaban incluidos los egresados de las escuelas normales nacionales y provinciales, así como quienes habían rendido en mesas examinadoras, realizado los cursos temporarios, 0 incluso sólo habían aprobado una materia y con ello tenían un certificado habilitante. El

\footnotetext{
${ }^{9}$ Fundado en 1898 por medio de la ley № 1669 dependía del Ministerio de Gobierno provincial. Tenía como objetivo la experimentación, la investigación y la estadística agrícola. Progresivamente fue ampliando funciones y estructura organizacional.
} 
REVISTA DE LA ESCUELA DE CIENCIAS DE LA EdUCACIÓN, AÑO 16, NRO. 15, VOL. 2, JULIO A DICIEMRE DE 2020. PÁGINAS 143-154. ISSN 2362-3349 (EN LÍNEA). LA FORMACIÓN DEL MAGISTERIO RURAL EN ENTRE RÍOS: DEMANDAS PRODUCTIVAS Y ASPIRACIONES PROFESIONALES (1914-1930). JANET PRISCILA CIAN

minucioso examen realizado a partir de los nuevos criterios de la administración educativa mostraba que, en el caso de los maestros provinciales, sólo 367 eran diplomados, en lugar de 714, con lo cual sólo un $43 \%$ del plantel docente provincial tendría título docente (Provincia de Entre Ríos, 1919, p. 13). Lo cual difería con el 83,41\% presentado por la estadística de 1913.

Aunque suprimidos con el recambio de autoridades gubernamentales, los cursos temporarios fueron rehabilitados en diciembre de 1915, por la solicitud de quienes no los habían terminado y por la elevada cantidad de maestros sin títulos que seguía ejerciendo en el ámbito rural. De este modo, se reeditaba el argumento que aludía al poco interés de los maestros normales por trabajar en el medio rural dada las dificultades del medio, la escasa remuneración y la insuficiencia de maestros rurales egresados con relación a las necesidades de la provincia (Provincia de Entre Ríos, 1919, p. 12). El 20 de octubre de 1915, el CGE dictó una resolución mediante la cual fijaba el mecanismo, período y límite de tiempo para la regularización de la situación de los maestros que no poseían título o solo tenían un certificado habilitante y se decretó caducos todos los certificados habilitantes otorgados previamente. Las mesas examinadoras funcionarían en enero y julio y los aspirantes que aprobaran el primer término debían presentarse inmediatamente al año siguiente para rendir el examen, con pena de perder el tramo realizado.

Para el caso de la Escuela Normal Rural, ese mismo año, fue repuesto en el cargo de director el profesor normal Amadeo Autcher, trasladado luego del sumario e intervención a la escuela efectuados en 1912. En la Memoria del CGE se señalaba el creciente prestigio de la escuela, que dio lugar al aumento de los aspirantes, pero que no pudieron ingresar por falta de infraestructura. También se destacaba la mejoría en la administración de los recursos, a pesar de las restricciones generales que afectaban todas las áreas, que había repercutido en la falta de algunas secciones productivas destinadas a la práctica agropecuaria ${ }^{10}$. Por falta de presupuesto, el plantel de profesores había sido reducido, tal como había ocurrido en las otras escuelas especiales, generando una recarga horaria para cada profesor. La escuela también realizaba una tarea de extensión mediante la distribución de plantas en el vecindario y tenía un importante plantel avícola que demandaba urgentes mejoras en las especies e instalaciones. En 1915 graduaron 16 maestros, la mayoría de los cuales se desempeñaban a las escuelas provinciales, a excepción de uno que continuaba sus estudios en una institución nacional.

El Censo Escolar de 1916 arrojó cifras polémicas con respecto a la estadística previa, especialmente en el número de analfabetos ${ }^{11}$. En lo referente al personal en ejercicio, las cifras aludían a un total de 696 maestros de las escuelas fiscales con diploma. Al año siguiente, la cantidad total de maestros diplomados era de 1.562 , de los cuales el $72,91 \%$ ejercía en las escuelas provinciales, el $79.91 \%$ en las nacionales, el $63.41 \%$ en las municipales y el $35.91 \%$ en las particulares (Provincia de Entre Ríos, 1919, p. 162). Con respecto al período anterior, se habían eliminado los maestros especiales y aquellos que cumplían otras tareas en la administración educativa. Para atender las dificultades del magisterio y estimular su desarrollo, algunos funcionarios educativos e inspectores demandaban al PE provincial el cumplimiento en el pago de haberes, la creación de un Banco Escolar y el establecimiento del escalafón docente que contemplara el aumento salarial progresivo y con lo cual se beneficiaría, especialmente, el personal docente que actuaba en las escuelas rurales

En 1917, a las altas tasas de analfabetismo y la carencia de recursos, producto de las consecuencias de la crisis económica general y dificultades locales climáticas, se sumaban los enfrentamientos políticos en la legislatura provincial $^{12}$ que trababan la actualización de la ley de presupuesto. Por este motivo, y ante la demanda de atención del analfabetismo escolar, el CGE creo "escuelas libres" y "escuelas de familia" ", alternativas menos institucionales, que insumían pocos recursos y permitían una escolarización básica de la población. Ese mismo año, el 27 de septiembre ${ }^{14}$, el presidente del CGE, Alfredo Villalba, elevó al Ministro de Hacienda, Justicia e Instrucción pública el "Programa de Ley sobre Estabilidad y aumento progresivo de sueldos del magisterio de la provincia”, una petición que venía siendo canalizada a través de la prensa local ${ }^{15}$.

\footnotetext{
${ }^{10}$ En mayo de ese año se restituyó el examen de ingreso para todos los aspirantes, algo que en la práctica estaban exceptuados los alumnos provenientes de las escuelas agropecuarias de la provincia.

${ }^{11}$ La realización del censo escolar provincial de 1916 arrojó las siguientes cifras: el $42.83 \%$ de los niños de 6 a 14 años no sabían leer y escribir, el $1,84 \%$ saben leer o escribir solamente y sólo el 55,33\% podían leer y escribir

${ }^{12}$ El 21 de septiembre de 1916 se dictó la ley de intervención de la provincia de Entre Ríos por parte del PEN. Recién se logró regularizar la situación el 23 de abril de1917, cuando el interventor nacional convocó a la Asamblea General para la elección de senadores nacionales.

${ }^{13}$ Se crearon mediante resolución del CGE el 11 de mayo de 1917. Funcionaban en el medio rural en la casa de la persona autorizada por el Consejo para su apertura. Se debía enseñar a leer, escribir y contar. Debían estar alejadas del radio de influencia de las escuelas fiscales y por cada alumno concurrente se le retribuiría con un peso.

${ }^{14}$ Desde la prensa local, opositora a la UCR, se señalaba "En pro del magisterio. Un interesante y tardío proyecto sobre estabilidad, sueldos y ascensos". La Acción, 27 de septiembre de 1917.

15 "El escalafón del magisterio ¿aptitudes o títulos? La Acción 22 de julio de 1915.
} 
Dicha propuesta fue tratada en la legislatura en 1918 y, el 9 de septiembre, fue sancionada la Ley № 2545 de estabilidad y escalafón del magisterio ${ }^{16}$, considerada por sus contemporáneos la primera en su género en el país (Reula, 1969). Entre los fundamentos para garantizar la estabilidad del magisterio y el mejoramiento salarial, la titulación era un aspecto central ${ }^{17}$. De esta forma, la nueva normativa presentaba la siguiente jerarquización: en primer lugar el título de profesor normal, luego el maestro egresado de la misma institución; seguía el maestro normal rural ${ }^{18}$, el maestro rural y, en última instancia, el maestro especial. Se establecía, además, que los títulos de sub preceptor normal y de maestro provincial equivalían a título de maestro normal rural. En la reglamentación de la ley, dispuesta meses después, se clarificó los requisitos para la obtención del título de maestro provincial y su equiparación al de maestro normal rural, con lo cual se facilitaba el acceso a una titulación, sin la necesidad de permanecer durante tres o cuatro años ${ }^{19} \operatorname{como}^{\circ}$ internos en la Escuela Normal "Juan Bautista Alberdi".

En la Escuela Normal Rural, la obra fundamental habría sido "encauzarla convenientemente para que su finalidad única fuera formar maestros rurales". Con esto se aludía a la reducción de lo que se consideraba secundario en la institución, un eufemismo que velaba los proyectos truncos de secciones productivas ${ }^{20}$, definidas como una carga inútil por representar mayor trabajo para el personal y, por sobre todo, “... una distracción de tiempo con evidente perjuicio para su capacidad docente futura" (Provincia de Entre Ríos, 1919, p. 188). Un aspecto que comenzaba a cobrar fuerza en el discurso de los directivos y de los funcionarios era la definición del perfil profesional del maestro normal rural, advirtiéndose que por la historia institucional de la escuela recayó sobre ellos una exigencia exagerada, que desvirtuó el objetivo central: la formación de maestros para el campo. Así lo expresaba su director:

...en los últimos años se ha tenido como objeto principal la formación de maestros, es decir, se ha querido que un alumno egresado no solo como el rutinario enseñador de la lectura, escritura y matemáticas elementales (leer, escribir y contar) sino que lleve un caudal de conocimientos que lo coloquen de lleno en la corriente de la evolución social y científica (...) esto ha debido ir en perjuicio de la preparación agropecuaria e industrial. Se empezó por suprimir la fábrica de hielo; luego la cremería- dos secciones montadas según los últimos adelantos de la industria- porque se pensó, con justicia, que el maestro no iría a enseñar en la campaña como se instala una fábrica de hielo o una cremería en grande escala; en la primera época los alumnos debían interrumpir sus estudio durante uno o dos meses para dedicarse a los trabajos de agricultura, arboricultura; ahora se estudia de mañana y se practica en el campo, en el jardín en el gallinero etc. por la tarde. De esta manera, cada faz de la enseñanza tiene según su importancia relativa para el pedagogo su estimulo teórico y la práctica de la enseñanza en la escuela de aplicación anexa; para el 'chacarero rural', la práctica en las distintas secciones de la escuela bajo la dirección de los jefes respectivos- actualmente las secciones son: agricultura general y especial, arboricultura, jardinería, horticultura, avicultura, apicultura, puericultura, tambo y cremería, taller... (Escuela "JBA" Epistolario 1915-1919, p. 310).

Hacia 1917, el resumen de la actividad de la escuela indicaba que habían egresado 151 maestros en once años. Del total de egresados, sólo se tenía registro de actividad profesional de 77 y habían fallecido 4, con lo cual casi la mitad de los egresados no ejercía la docencia. No obstante, la necesidad de formalizar los cambios que veían registrándose en las prácticas de formación de los maestros rurales derivó en la conformación de una comisión encargada para de la tarea. El 16 de enero de 1920 se dictó la resolución para la organización y sus funciones, quedando constituida por los inspectores Esteban Bugnone y Lucio Macedo, junto con el director del establecimiento, Carlos Bravo. Su misión era reformar el plan de estudio y el

\footnotetext{
${ }^{16}$ Reglamentada el 5 de diciembre de 1918.

${ }^{17}$ Se establecía que los nombramientos del personal docente, directivo y de inspección de las escuelas públicas se harán "en riguroso orden de mérito determinado por la categoría del título, el concepto personal y los servicios profesionales prestados en el país" (Art. 1) y eran inamovibles mientras tengan buena conducta. Los maestros sin título eran interinos y tenían una remuneración menor a los titulados. Cuando obtenían su titulación, se les reconocía la antigüedad en el cargo.

${ }^{18}$ También se podía acceder al título de maestro normal rural con dos años de buenos servicios y el cursado de $3^{\circ}$ año en la Escuela "Alberdi".

${ }^{19}$ Se había implementado el curso preparatorio en la escuela de aplicación anexa, donde los jóvenes aspirantes al magisterio podían cursar el 6 año requerido para el ingreso.

${ }^{20}$ Se seguía brindando una beca a los alumnos distinguidos de las escuelas elementales con anexo agropecuario para que continuaran estudios en Alberdi.
} 
REVISTA DE LA ESCUELA DE CIENCIAS DE LA EDUCACIÓN, AÑo 16, NRO. 15, VOL. 2, JULIO A DICIEMRE DE 2020. PÁGINAS 143-154. ISSN 2362-3349 (EN LÍNEA). LA FORMACIÓN DEL MAGISTERIO RURAL EN ENTRE RÍOS: DEMANDAS PRODUCTIVAS Y ASPIRACIONES PROFESIONALES (1914-1930). JANET PRISCILA CIAN

reglamento interno. Los estudios agrícolas y ganaderos debían ser los indispensables, considerando el carácter anexo que tenían en el establecimiento Normal Rural. Por ello, se recomendaban especialmente los estudios pedagógicos “... y dentro de ellos los de la metodología general especial y práctica de la enseñanza, las matemáticas, el lenguaje -con especialidad composición, lectura y ortografía-y la caligrafía" (Provincia de Entre Ríos, 1920, p 16). En el caso de la formación agropecuaria, se establecía que los alumnos realizarían prácticas periódicas y por turno en las distintas secciones, en huertas y jardines. Se mantenía la misma duración del plan de estudios - de tres años- y como requisito de ingreso se debía tener aprobado, el quinto grado de escolaridad primaria.

El informe de la comisión señalaba que las reformas se orientaron a la finalidad práctica que debía brindar la escuela. En cuanto al funcionamiento interno, se había tendido hacia cierta descentralización de las diversas secciones productivas, regulando las atribuciones de cada jefe de sección. De esta forma el director podía estar al tanto del funcionamiento de la escuela en su faz instructiva, práctica, económica y educativa. Las reformas introducían un control administrativo que otorgaba a la escuela mayor autonomía en el manejo de los fondos producidos. En lo referente los alumnos, se señalaba específicamente su condición de alumnosmaestros. En el plan de estudios se daba mayor amplitud a las asignaturas para la formación nacional y para la formación y práctica docente. Los programas de las materias debían ser guías y "... no la enunciación de principios científicos o de conceptos abstractos...", realzando los cursos de castellano y de historia porque:

...en una provincia como la de Entre Ríos, que recibe inmigrantes de diferentes nacionalidades, muchos de ellos rebeldes a nuestra organización política y desenvolvimiento social, la enseñanza de la historia debe contribuir, ante todo, a mantener íntegramente el carácter nacional, ajustado a la liberalidad de nuestras leyes y atendiendo que tiende al mismo fin la enseñanza del idioma, de la geografía e Instrucción Cívica (Provincia de Entre Ríos, Boletín de Educación, 1920, p. 16).

El trabajo de la comisión se aprobó con algunas modificaciones al plan de estudio y a la distribución de la carga horaria, componiéndose de: Enseñanza Profesional (pedagogía, práctica de la enseñanza), Enseñanza General (aritmética, geometría, castellano, geografía, historia, instrucción moral y cívica, ciencias naturales, física, química, música y canto) y Enseñanza Industrial, compuesta por prácticas agropecuarias y de taller (Figura I).

En lo referente al funcionamiento de los cursos temporarios, las estadísticas difundidas mostraban una progresión en la cantidad de aprobados ${ }^{21}$. Sin embargo, fue necesario instituir algunos cambios, entre ellos imponer restricciones en el otorgamiento de pasajes, alojamiento y viáticos, por medio de una resolución de CGE del 6 de noviembre de 1919. Los maestros en ejercicio sólo disfrutaran de alojamiento y pensión en la escuela Alberdi en un número reducido y los aspirantes -tanto varones como mujeres- que fueran interinos en sus cargos podían solicitar pasajes oficiales a descontar de sus remuneraciones. Por resolución del 28 de septiembre de 1920, los cursos temporarios fueron restablecidos con nueva organización y programas (Provincia de Entre Ríos, 1922) que incluía pedagogía y práctica, aritmética, geometría, castellano, geografía, historia, ciencias naturales, instrucción moral y cívica, dibujo, música y manualidades (mujeres) o bien industrias rurales (varones). También se dictó la ley № 2.746 que incluyó la disposición de otorgar, por única vez, el título de maestro rural a los docentes que tenían más de diez años de servicio y buen concepto en las escuelas rurales. También quienes contaban con dos años de ejercicio docente conceptuado positivamente podían rendir examen y obtener el título de maestro provincial, análogo al título de maestro normal rural. Según la estadística escolar publicada en el Boletín 1920, la cantidad de maestros diplomados de las escuelas provinciales eran: el $72,62 \%$ en 1917 , el $78 \%$ en 1918 , y el $85 \%$ en $1919^{22}$.

\footnotetext{
${ }^{21}$ En el período 1916-17 concurrieron 47 aspirantes y aprobaron el curso 13 (27, 5\%). En 1917-18 concurrieron 42 y aprobaron 26 (61, 60\%). En 1918-19 concurrieron 29 y aprobaron 22 (75, 86\%). Fuente: La Acción, 18 de febrero de 1919. ${ }^{22}$ En las escuelas nacionales la proporción eran de 79\% 91\% 85, $5 \%$ y 87, 3\% .En municipales 63\%, 41\%, 63, $9 \%$ y 56\%. En las particulares $35 \%, 91 \%, 21 \%$, y $25 \%$.
} 
REVISTA DE LA ESCUELA DE CIENCIAS DE LA EdUCACIÓN, AÑO 16, NRO. 15, VOL. 2, JULIO A DICIEMRE DE 2020. PÁGINAS 143-154. ISSN 2362-3349 (EN LÍNEA). LA FORMACIÓN DEL MAGISTERIO RURAL EN ENTRE RÍOS: DEMANDAS PRODUCTIVAS Y ASPIRACIONES PROFESIONALES (1914-1930). JANET PRISCILA CIAN

Figura I: Planes de estudio y carga horaria de materias Escuela Normal Rural “JB Alberdi”

\begin{tabular}{|c|c|c|c|c|c|c|}
\hline AÑOS & & 19 & & \multicolumn{3}{|c|}{1920} \\
\hline Asignaturas hs & $1^{\circ}$ & $2^{\circ}$ & 3은 & 19 & $2^{\circ}$ & 3은 \\
\hline Pedagogía & 3 & 3 & 2 & 3 & 3 & 3 \\
\hline Aritmética & 2 & 2 & 1 & 3 & 2 & 2 \\
\hline Geometría & 2 & 1 & 1 & 2 & 2 & 2 \\
\hline Historia & 2 & 2 & 2 & 3 & 2 & 2 \\
\hline Geografía & 2 & 2 & 2 & 2 & 2 & 2 \\
\hline $\begin{array}{l}\text { Idioma } \\
\text { nacional }\end{array}$ & 5 & 4 & 3 & & & \\
\hline Geología & 1 & & & & & \\
\hline Mineralogía & 2 & & & & & \\
\hline Agricultura & 1 & 1 & 1 & 1 & 1 & 1 \\
\hline Física & 1 & 1 & & & 2 & 1 \\
\hline Química & 1 & 1 & 2 & & 1 & 1 \\
\hline $\begin{array}{l}\text { Industrias } \\
\text { rurales }\end{array}$ & & 2 & 2 & 1 & 1 & 1 \\
\hline Botánica & & 1 & & & & \\
\hline Zoología & & 1 & & & & \\
\hline Zootecnia & & & 2 & & & 1 \\
\hline $\begin{array}{l}\text { Instrucción } \\
\text { cívica (moral) }\end{array}$ & & & 2 & & & 2 \\
\hline $\begin{array}{l}\text { Anatomía y } \\
\text { fisiología }\end{array}$ & & & 2 & & & \\
\hline Dibujo & 2 & 1 & 1 & 1 & 1 & 1 \\
\hline Caligrafía & 1 & 1 & & & & \\
\hline $\begin{array}{l}\text { Observación, } \\
\text { critica y } \\
\text { practica de la } \\
\text { enseñanza }\end{array}$ & 3 & 6 & 6 & 3 & 4 & 5 \\
\hline $\begin{array}{l}\text { Música** y } \\
\text { canto }\end{array}$ & 2 & 1 & 1 & 1 & 1 & 1 \\
\hline Castellano & & & & 7 & 6 & 4 \\
\hline $\begin{array}{l}\text { Ciencias } \\
\text { Naturales }\end{array}$ & & & & 1 & 2 & 2 \\
\hline
\end{tabular}

Fuente: Elaboración propia en base a datos de Epistolario 1915-1919 de Escuela Juan Bautista Alberdi y Boletín de Educación, 1920.

* Se agregó en el plan de estudios vigente, el siguiente programa de Economía Política en 2ํ y 3ํ․ Incluía contenidos de cooperación y mutualismo, aspectos legales, función económica social. Tipos de cooperativas, subclases y especialmente cooperativas y bancos agrícolas

** En 1917 sólo se dictaba Música.

\section{Exceso de maestros, analfabetismo y obligatoriedad escolar}

El 1 de octubre de 1922 asumió la gobernación el radical Ramón Mihura ${ }^{23}$ y durante su gestión se registraron algunas tensiones con la recientemente organizada Asociación del Magisterio en torno al atraso de sueldos, la falta de cumplimiento de lo establecido en la Ley de escalafón y la disconformidad con la designación de un ingeniero como presidente del CGE. Un diagnóstico que comenzó a cobrar mayor fuerza era el de la existencia de un exceso de maestros, situación que se magnificaba en distintas notas de la prensa local, aunque ya había comenzado a manifestarse en la década anterior. Como consecuencia, el CGE

\footnotetext{
${ }^{23}$ En 1922 dos sectores del radicalismo se enfrentaron por la candidatura a la presidencia nacional. Una llevó a Marcelo Torcuato de Alvear y la UCR principista al entrerriano Miguel Laurencena, ex gobernador de la provincia.
} 
REVISTA DE LA ESCUELA DE CIENCIAS DE LA EdUCACIÓN, AÑO 16, NRO. 15, VOL. 2, JULIO A DICIEMRE DE 2020. PÁGINAS 143-154. ISSN 2362-3349 (EN LÍNEA). LA FORMACIÓN DEL MAGISTERIO RURAL EN ENTRE RÍOS: DEMANDAS PRODUCTIVAS Y ASPIRACIONES PROFESIONALES (1914-1930). JANET PRISCILA CIAN

suprimió los cursos temporarios el 5 de octubre, fundándose en que sólo había 42 maestros sin título, de los cuales 7 tenían aprobados el primer año del curso y 11 debían las pruebas complementarias. No obstante, había 200 maestros normales inscriptos en el registro de aspirantes a cubrir cargos, 3 rurales, 17 con $1^{\circ}$ término y 18 profesores especiales. Esto provocaba un exceso de oferta de docentes que no tenían ubicación. Haciendo uso de la facultad discrecional del PE provincial para la apertura de exámenes, se consideró que sólo debía funcionar lo indispensable. Por eso se suprimieron los cursos temporarios, pero se mantuvieron las mesas examinadoras para: a) los maestros rurales que deseaban aspirar al título de maestro provincial; b) los maestros rurales que debían materias de $1^{\circ}$ o $2^{\circ}$ curso; c) para los maestros interinos o que tenían aprobado el $5^{\circ}$ grado y que deseaban rendir el $1^{\circ}$ termino. Desde la prensa, se describía la situación de este modo:

...cuatro mil maestros merodeaban los consejos escolares de Buenos Aires reclamando un puesto y la materia educativa no se preocupó mayormente por ello a pesar de la grave cuestión de estado que entrañaba. En esta provincia el exceso de docentes ha repercutido también provocando una resolución de la autoridad escolar por la cual se suspenden estos años los cursos temporarios con los que se combatió el maestro sin título con los resultados de todos conocidos. Todo ello quiere decir, que aquí en Entre Ríos hay más maestros de los que requiere el estado para confiarles la enseñanza pública y como el presupuesto es, por hoy, el único aliciente del gremio, resulta que el sacrificio de toda una carrera carece de compensación... (El Diario, 7/10/1923)

La falta de acuerdos políticos en el Senado de la provincia durante toda la gestión impidió la aprobación de la nueva ley de presupuesto, con lo cual los aumentos establecidos por Ley de Escalafón se habían cumplido en una minina proporción y cuya consecuencias, según los funcionarios locales, era la reducción de la cantidad de maestros varones en ejercicio (Provincia de Entre Ríos, 1926, p. 584). El aparente exceso de maestros contrastaba con los índices de analfabetismo que, en 1924, seguían siendo importantes $^{24}$, y eran considerados un problema que afectaba el desarrollo de la democracia, ya que con el escaso tiempo que los niños permanecían en las escuelas apenas podían acceder a algunos saberes elementales y por ello se los definía como "analfabetos intelectuales". Ese año se calculaba una proporción del $20 \%$ de analfabetos con relación a la población escolar. Entre las causas, se destacaba el medio ambiente y la ocupación del niño. En cuanto al primer punto, se reproducían argumentos de larga data en torno al vínculo entre actividad ganadera y población criolla, que se diferenciaba de la afincada en centros agrícolas, más propensa a la instrucción. Para resolver la situación se presentó un proyecto para garantizar la obligatoriedad escolar mínima, se instituyen libretas para este fin y ese mismo año se creó el cargo de maestro ambulante para escuelas de las zonas ganaderas del norte entrerriano. El problema del analfabetismo que afectaba, especialmente, a la población rural resultaba una consecuencia de las dificultades de la expansión de la escuela primaria, pero también el producto de las aspiraciones y necesidades de una población considerada refractaria a las potencialidades de la escolarización.

En lo referente al desarrollo institucional de la escuela "Alberdi", el 10 de noviembre de 1922 fue suspendido el director de la escuela Carlos Bravo y en su lugar asumió Juan Carponi Flores, quien se desempeñó durante toda la década. Ese año egresaron 12 maestros y al año siguiente lo hicieron 17; se desarrolló una exhaustiva planificación de las actividades en cada una de las secciones productivas -chacra, huerta, arboricultura, jardín, porqueriza, gallinero, cabañas, tambo, ganadería, cremería y trabajos generales-. La actividad productiva de la escuela continuaba reducida, ya que del total de 374 hectáreas que tenía en ese momento, 240 eran arrendadas a particulares y en el resto se desenvolvían las actividades de las secciones. Cada sección era atendida por los alumnos, distribuidos y organizados en grupo y según los años de cursado. Así, los aspirantes de $1^{\circ}$ año atendían y trabajaban en huerta, jardín, gallinero; los de $2^{\circ}$ en huerta y chacra; y los alumnos del último año debían hacerlo en chacra, arboricultura, tambo y porqueriza. En los talleres los alumnos practicaban pinturería, herrería y reparación de implementos agrícolas que colaboraban en el mantenimiento del establecimiento escolar. La tasa de egreso se mantuvo casi similar al año 1923, al culminar 13 jóvenes. En 1925, finalizaron sus estudios 10 maestros y en diciembre ${ }^{25}$ de ese año se registraron pedidos de becas para jóvenes paraguayos que deseaban cursar sus estudios en la escuela Alberdi, con la intermediación realizada por el cónsul de ese país.

\footnotetext{
${ }^{24}$ En una nota que dirigió el Comando de la III División del Ejército al PE provincial se señalaba que el 34\% de la clase militar que se incorporó ese año era analfabeto.

${ }^{25}$ El 2 de enero de 1925 se inauguró en la escuela "Alberdi" la primera colonia de vacaciones de la provincia a cargo de la Sociedad Amigos de la educación.
} 
En 1927, durante la gobernación de Eduardo Laurencena, se comenzaron a manifestar los primeros signos de la crisis agrícola y económica general que se profundizaría en los años siguientes. La oposición en la cámara de senadores y diputados derivó en el presupuesto fuese el mismo de 1923, afectando en particular al CGE que se manejó en déficit y no alcanzó a cubrir las necesidades de la expansión escolar. En 1928, la Escuela Normal Rural tenía 48 alumnos inscriptos en el curso normal y 130 en la escuela de aplicación. Del total, 89 estaban en condición de internos -41 becados y 48 pensionistas- y ese año egresaron 12 maestros normales rurales ${ }^{26}$. A año siguiente, la Escuela "Juan Bautista Alberdi" festejaba los 25 años de su apertura, celebración que concitó la atención y concurrencia de la prensa y las principales autoridades políticas de la provincia.

\section{Conclusiones}

El análisis realizado permite constar que existió cierta continuidad en el tratamiento del problema de la capacitación del magisterio rural, mediante el sostenimiento de las políticas e instituciones que habían sido creadas con anterioridad. Sin embargo, existen algunos elementos que establecen algunas diferencias con la etapa previa y que responden a una progresiva reorientación de los objetivos de la estatalidad provincial y las políticas públicas para la modernización del medio rural. El optimismo atribuido a la creación de instituciones escolares para el mejoramiento económico se fue transformando en un cierto pragmatismo, ya que la continuidad institucional podía resolver, parcialmente, el problema de la falta de maestros diplomados. En este contexto, la obtención de diploma se convertía en un punto clave ante la progresiva profesionalización del magisterio, mediante la jerarquización y escalafonamiento establecida por ley en esos años, aunque su efectivización estuvo mediada por las dificultades presupuestarias que condicionaron los sucesivos gobiernos. En este punto también interesa señalar que dentro de la organización jerárquica efectuada en la normativa que regulaba la tarea docente, los egresados de la Normal Rural veían fácilmente equipados sus títulos con aquellos que rendían exámenes para obtener el título de maestro provincial. Vinculado a esto, se puede conjeturar que parte de los cambios curriculares y en las prácticas de formación de los aspirantes de la Normal Rural, tendientes a consolidar una preparación profesional donde la especificidad rural ocupaba un espacio menor

La supresión de los cursos temporarios puede entenderse, ante todo, una medida de carácter económico. Esto se sustenta en la continuidad que tuvieron las mesas examinadoras para la obtención de titulo, la revalidación y también el examen que podían dar quienes tenían 5 grado acreditado para la obtención del título de maestro rural. Si bien la ausencia de la capacitación de los cursos temporario puede haber generado dificultades en los aspirantes, la posibilidad de acceso al título de magisterio rural permanecía. El argumento de exceso de maestros y la falta de plazas para cubrir parecía soslayar el problema de la expansión de la escuela en el medio rural, con la consecuente demanda presupuestaria que exigía -infraestructura, insumos y personal-. Los intentos de escuelas de familia, maestros ambulantes y los mecanismos para asegurar la obligatoriedad escolar pueden considerarse paliativos de un problema mayor, alejado de las posibilidades presupuestarias de los gobiernos de la UCR, afectado por las crisis económicas del período y las dificultades políticas, amplificadas por la disputa intrapartidaria local.

\section{Referencias bibliográficas}

Ascolani, A. (2017). Concepciones reformistas en torno a las funciones de la Educación Primaria Rural Argentina (1930-1960). En: Dialogia, São Paulo, no 25, enero-abril 2017, pp. 43-68. Recuperado de http://periodicos.uninove.br/index.php?journal=dialogia\&page=article\&op=view\&path\%5B\%5D=6997

Ascolani, A. (2012). La escuela primaria rural en Argentina. Expansión, orientaciones y dificultades (1916-1932). En: Teias, 14(28), pp. 309-324. Recuperado de: http://www.epublicacoes.uerj.br/index.php/revistateias/article/view/24245

\footnotetext{
${ }^{26}$ Se señalaba que desde 1907 hasta el año pasado, se han graduado 267 maestros normales rurales.
} 
REVISTA DE LA ESCUELA DE CIENCIAS DE LA EDUCACIÓN, AÑo 16, NRO. 15, VOL. 2, JULIO A DICIEMRE DE 2020. PÁGINAS 143-154. ISSN 2362-3349 (EN LÍNEA). LA FORMACIÓN DEL MAGISTERIO RURAL EN ENTRE RÍOS: DEMANDAS PRODUCTIVAS Y ASPIRACIONES PROFESIONALES (1914-1930). JANET PRISCILA CIAN

Ascolani, A. (2015). Ruralidad, analfabetismo y trabajo en la Argentina. Proyectos y acciones del Consejo Nacional de Educación (1930-1940). En: Cadernos de História da Educação 14(3), pp. 853-877. Recuperado de: http://www.seer.ufu.br/index.php/che/article/view/33141

Ascolani, A. (2007). Las Escuelas Normales Rurales en Argentina. Una transición entre las aspiraciones de la cultura letrada el imaginario de cambio socioeconómico agrario (1900-1946). En: Corrêa Werle, F. (comp.). Educação Rural em Perspectiva Internacional. Instituições, práticas e formação do professor (pp. 373-424). ljuí, Brasil: Editora UNIJUI.

Barsky, O. y Gelman, J. (2005). Historia Del agro argentino. Desde La conquista hasta fines del siglo XX. Buenos Aires: Mondadori.

Bohoslavsky, E. y Soprano, G. (2010). Un Estado con rostro humano. Funcionarios e instituciones estatales en Argentina (de 1880 a la actualidad). Buenos Aires: Universidad Nacional de General Sarmiento y Prometeo Libros.

Carli, S. (1993). Modernidad, diversidad cultural y democracia en la historia educativa entrerriana (1883-1930). En: Puiggros, A. (Dir.). La educación en las provincias y territorios nacionales (1885-1945). Historia de la educación en la Argentina IV, pp185-237. Buenos Aires: Galerna.

Cattaruzza, A (2016). Historia de la Argentina 1916-1955. Buenos Aires: Siglo XXI.

Cian, J. P (2018). Orígenes de las escuelas agropecuarias en la provincia de Entre Ríos, Argentina 1896-1910. En: Mundo Agrario Vol. 19 (№ 42) pp. 1-17. Universidad Nacional de La Plata. Disponible en: https://www.mundoagrario.unlp.edu.ar/article/view/MAe093

Cattaruzza, A. (2019). Instituciones educativas para el desarrollo de la vitivinicultura litoral. Entre Ríos, Argentina (1904-1918). En: RIVAR Vol. 6, (№ 17), p 90-111.

Devoto, F. (2009). Historia de la inmigración en la Argentina. Tercera Edición, Buenos Aires: Sudamericana.

Di Liscia, M.S y Soprano, G. (2017). Burocracias estatales. Problemas, enfoques y estudios de caso en la Argentina (entre fines del siglo XIX y XX). Rosario: Prohistoria.

Fiorucci, F. (2014). Maestros para el sistema de educación pública. La fundación de escuelas normales en Argentina (1890-1930). En: Revista Mexicana de Historia de la Educación, vol. II, núm. 3, 25, pp. 25-45. Recuperado de: http://ri.conicet.gov.ar/bitstream/handle/11336/35527/CONICET_Digital_Nro.08b2aae6-1c72-4ef2-9c321a0596fb631b_A.pdf?sequence=2\&isAllowed =y

Gutiérrez, T. (2007a). Educación, agro y sociedad. Políticas educativas agrarias en la región pampeana 1897-1955. Buenos Aires: Editorial Universidad Nacional de Quilmes.

Gutiérrez, T. (2007b). Políticas de orientación agrícola y pedagogía normalista. Entre Ríos, Argentina, 1900-1920. Perfiles Educativos, 29(117), pp. 85-110. Universidad Nacional autónoma de México. Recuperado de: http://www.redalyc.org/articulo.oa?id=13211705

Hora, R. (2010). Historia económica de la Argentina en el siglo XIX. Buenos Aires: Fondo de Cultura Económica.

Mayer, M. S. (2014). Educación rural, inmigración y relaciones sociales. Dos procesos de colonización agrícola en la provincia de Entre Ríos. Buenos Aires: la Colmena.

Mateo, J., et. al. (2017). Poblamiento, despoblamiento y repoblamiento de la provincia de Entre Ríos. Un ensayo de demografía histórica (1869-2010). En: EJES de Economía y Sociedad, 1(1), 75-94.

Míguez, E. (2012). Historia económica de la Argentina: De la conquista a la crisis de 1930. Buenos Aires: Sudamericana.

López, M. P. (1999). La educación de rusos judíos y alemanes del Volga en Entre Ríos. Conflictos políticopedagógicos (1880-1910). En Ascolani, A. (comp.), La educación en Argentina. Estudios de historia (pp 71 83). Rosario: Ediciones del Arca.

Ossanna, E y Hadad, M (2011). La educación en números. Los aportes y los conflictos de la estadística educativa: varios intentos de lectura. En: Ossanna et. Al. Quienes leen, qué leen y cómo leen los entrerrianos en las últimas décadas del siglo XIX y primeras décadas del siglo XX. Vice gobernación, FCE.

Palermo, S. y Silva J. (2016). Expertos, burocracias y política de masas en Argentina. En: Estudios Sociales del Estado V 2, № $\quad 3, \quad$ pp. 6-21. Recuperado de: http://estudiossocialesdelestado.org/index.php/ese/article/view/74

Petitti, M. E. (2016). La educación primaria en los campos de la provincia de Buenos Aires (1943-1955). Mundo Agrario, V 17, № 34, pp. 1-23 Recuperado de: https://www.mundoagrario.unlp.edu.ar/article/v

Pierini, M.A (2016). La educación rural desde la perspectiva de una historia regional de la Patagonia Austral. En:

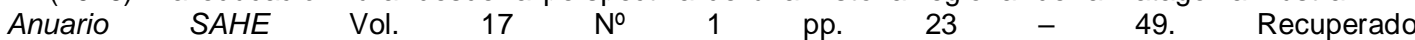
http://ppct.caicyt.gov.ar/index.php/anuario/article/view/8662/pdf

Provincia de Entre Ríos (1932). Memoria del Ministerio de Hacienda, Justicia, Culto e instrucción pública de Entre Ríos correspondiente al periodo comprendido dese el $1^{\circ}$ de octubre de 1930 hasta el 29 de diciembre de 1931. Paraná: El Diario.

Provincia de Entre Ríos (1919). Memoria de la Dirección General de Escuelas de Entre Ríos correspondiente a los años 1914, 1915, 1916 y 1917. Paraná: Publicación oficial.

Provincia de Entre Ríos (1920). Boletín de Educación 5época, № 41 y 2 trimestre. Paraná: publicación oficial.

Provincia de Entre Ríos (1922). Consejo General de Educación. Síntesis de la obra realizada desde el 1ํde octubre de 1918 al 31 de marzo de 1922. Paraná: Publicación oficial. 
REVISTA DE LA ESCUELA DE CIENCIAS DE LA EdUCACIÓN, AÑO 16, NRO. 15, VOL. 2, JULIO A DICIEMRE DE 2020. PÁGINAS 143-154. ISSN 2362-3349 (EN LÍNEA). LA FORMACIÓN DEL MAGISTERIO RURAL EN ENTRE RÍOS: DEMANDAS PRODUCTIVAS Y ASPIRACIONES PROFESIONALES (1914-1930). JANET PRISCILA

CIAN.

Provincia de Entre Ríos (1926). Memoria de los Ministerio de Gobierno, Hacienda, Justicia e Instrucción Pública 1922-1926. Paraná: imprenta oficial

Provincia de Entre Ríos (1919). Escuela Juan Bautista Alberdi, Epistolario 1915-1919.Archivo institucional-museo escolar.

Regalsky, A. M. (1986). Las inversiones extranjeras en la Argentina, 1860-1914. Buenos Aires: Centro Editor de América Latina.

Reula, F. (1969). Historia de Entre Ríos. Santa Fe: Castellví.

Pereyra, E. (2012). El Estado y la Administración Pública nacional en perspectiva histórica. Análisis crítico de la producción académica sobre el período 1930-1976. En: Polhis, Vol. 5, № 9, pp. 92-112. 